\title{
Design of a Dummy Load for the 2 MW HF Transmitter
}

\author{
Jong-Gu Kwak ${ }^{*} \quad$ Non-member \\ Song-Jong Wang* Non-member \\ Jae-Sung Yoon* Non-member \\ Bong-Guen Hong* Non-member
}

\begin{abstract}
A $2 \mathrm{MW}$ transmitter with a frequency ranging from 30 to $60 \mathrm{MHz}$ is used for the KSTAR ICRF heating source and it is necessary to equip it with a dummy load which has a low VSWR above the second or third harmonic frequency for testing the $2 \mathrm{MW}$ transmitter. The resistive film type is well known as a non-inductive dummy load, however it can't be used above $300 \mathrm{~kW}$ because of problem at the surface adhesion between the ceramic substrate and the resistive film or sputtered carbon which imposes a limit on the power density of the film resistor. So, above $1 \mathrm{MW}$ the most promising candidate is the soda water dummy load where the soda water plays a role in both the rf power absorbing medium and the cooling water. In this work, the electrical and mechanical design requirements of the $2 \mathrm{MW}$ soda dummy load are introduced and RF characteristics are discussed using a lumped circuit model and a numerical analysis.
\end{abstract}

Keywords: transmitter, dummy-load, soda

\section{Introduction}

A RF power source with a frequency ranging around $50 \mathrm{MHz}$ is widely used in broadcasting and plasma heating applications. Especially, in the thermo-nuclear fusion experiments, the ICRF (Ion Cyclotron Resonant Frequency) is located around the HF frequency and a high power HF transmitter is required to achieve effective fusion yields. For the ITER (International Thermonuclear Experimental Reactor) case, the RF power of the unit transmitter is $2 \mathrm{MW}$ and 10 sets of $2 \mathrm{MW}$ transmitters are used. It is necessary to equip it with a dummy load for the RF power test of the transmitter. Generally, the resistive film type dummy load is widely used, however, it has a limit for an application above 300 $\mathrm{kW}$ because there is an adhesion problem between the resistive film material and ceramic substrate at a high temperature. Therefore, the most promising candidate above $2 \mathrm{MW}$ is the soda water dummy load where the soda water is used for both a resistive material and a cooling medium. In addition, there is no power density limit for the soda dummy load if it is operated below the boiling point of water.

In this work, the electrical and mechanical design requirements of the $2 \mathrm{MW}$ soda dummy load for the KSTAR (Korea Superconducting Tokamak Advanced Research) ICH (Ion Cyclotron Heating) system are introduced and discussed using a lumped circuit model and numerical analysis. The minimum requirement for a dummy load is that it has a heat capacity of $2 \mathrm{MW}$ and the VSWR is less than 1.5 up to $150 \mathrm{MHz}$.

\footnotetext{
* Korea Atomic Energy Research Institute

150, Dukjin, Yuseong, Daejeon, Korea
}

\section{General Description of the RF Dummy Load}

Fig. 1 (a) shows the resistive film type dummy load. It is composed of three sections. One is the first inner cylinder (I) where most of the rf power is dissipated on a very thin layer of resistive film coated onto the ceramic substrate. Another is the second inner cylinder (II) for

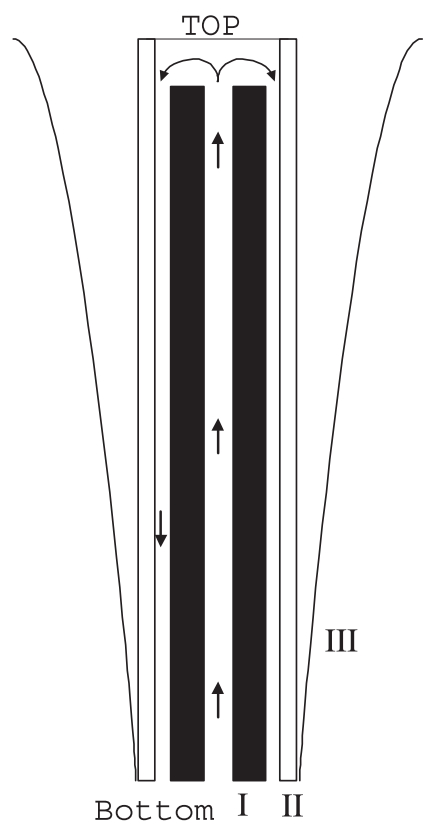

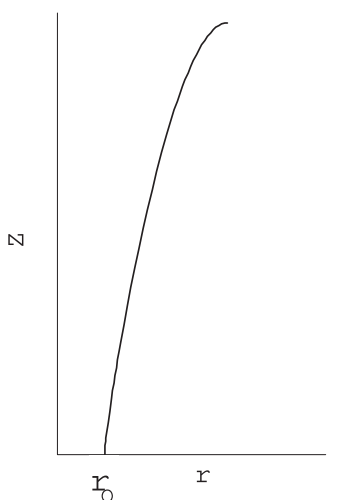

(b) (a)

Fig. 1. Schematic of typical resistive film type dummy load (a) and the profile function of section III (b) 
the closure of the coolant medium. The last one is the outer enclosure (III) with the shape of a conical as described in Fig 1 (b). The rf current of the center conductor is shorted to the ground at the bottom. The cooling water flows into the bottom of the first inner cylinder and then it flows out from the bottom between the first and second cylinder. The shape of the outer conductor (III) is important because it mainly determines the VSWR (Voltage Standing Wave Ratio) characteristics of the dummy load. If we assume that the resistance is proportional to the distance from the short point, for a perfect matching of the rf power to the dummy load, the characteristic impedance, $Z_{0}$ should be

$$
Z_{0}(z)=Z_{T 0}(1-z)
$$

where $Z_{T 0}$ is the characteristic impedance of the main transmission line which is the same as the termination resistance and it is typically $50 \Omega$. So the shape function of the outer conductor, $r(z)$ is

$$
r(z)=r_{0} e^{Z_{0}(z) / 60}
$$

where $r_{0}$ is the outer radius of the inner conductor (I). The only difference between the soda and resistive type dummy load is that the resistive film is replaced by the soda water which flows between the two conical cylinders. For the soda water dummy load, the water plays a role in both the cooling and rf power absorbing medium. As the resistive soda water also flows into the inner part of the dummy load, the skin depth effect should be considered. The skin depth is defined by

$$
\delta=\frac{1}{\sqrt{f \mu \sigma}}
$$

where $f$ is the RF frequency, $\mu$ is the magnetic permeability and $\sigma$ is the conductivity. The conductivity is given by

$$
\sigma=\frac{L}{R_{d c} A}
$$

for a cylinder whose DC resistance is $R_{d c}$, the area is $A$ and the length is $L$. For the resistive dummy load, the skin depth is larger than the thickness of the resistive film and the resistance value is independent of the frequency. However the resistance is a function of the frequency for a soda dummy load because AC resistance of the soda water varies as a function of the skin depth ${ }^{(1)}$ given by

$$
R_{a c}=\frac{R^{2}}{R^{2}-(R-\delta)^{2}} R_{d c} \cdots \cdots \cdots \cdots \cdots \cdots \cdots
$$

where $R$ is the radius of the soda water column. The skin depth is decreased and the AC resistivity becomes smaller as the frequency is increased. Therefore the VSWR becomes worse as the frequency is increased.

\section{Lumped Electric Circuit Model and a Numerical Analysis}

The soda water dummy load can be modeled by a tapered transmission line model ${ }^{(2)}$. When we consider

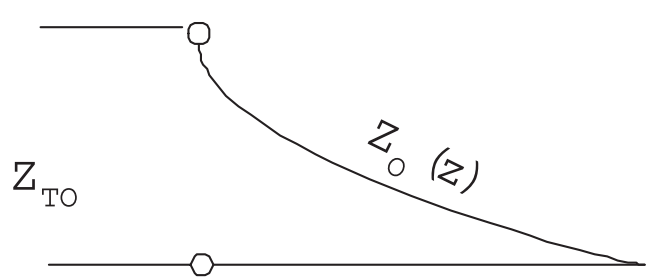

(a)

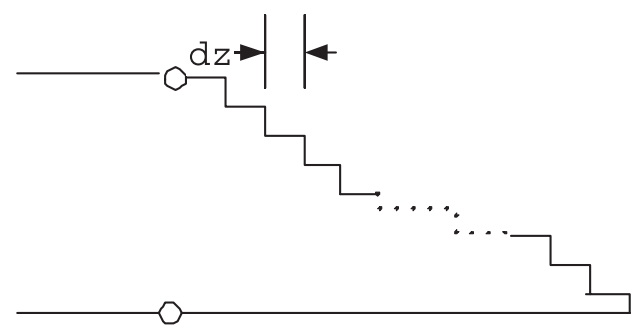

(b)

Fig. 2. Tapered transmission line (a) and its $\mathrm{N}$ discrete multi section equivalent (b)

the circuit as a tapered transition whose characteristic impedance varies continuously and smoothly from $Z_{T 0}$ to zero, it is replaced by the multi section transformer symbolized by Fig. 2 (b) and consisting of $\mathrm{N}$ discrete steps of length $L$. It is assumed that the resistivity varies in Eq. 5. The input impedance at the input port of each section is given by

$$
Z_{a c}=Z_{S 0} \frac{Z_{L} \cosh \gamma l+Z_{S 0} \sinh \gamma l}{Z_{S 0} \cosh \gamma l+Z_{L} \sinh \gamma l} \ldots \ldots \ldots
$$

where $\gamma$ is the propagation constant, $Z_{L}$ is the load impedance and $Z_{S 0}$ is the characteristic impedance for each section. From Eq. (6), the VSWR can be easily deduced for a nominal characteristic impedance of a transmission line. Fig. 3 shows various models of a soda water dummy load for a lumped circuit model and a numerical analysis. Depending on the shape of the outer conductor and the portion of the water filled volume in the inner conductor, the model can be categorized as four individual models. Model (a) and (c) describe the actual soda dummy load more than model (b) and (d) because the inner conductor has to be full with water to have a path for water cooling channel. The outer diameter of the input port is $345.2 \mathrm{~mm}$ and the length of the column is $500 \mathrm{~mm}$. Fig. 4 shows the typical characteristic of the VSWR of the soda dummy load using the lumped circuit model. The thickness of the water column is $15 \mathrm{~mm}$. It is assumed that cylinder (I) consists of air and its radius is $75 \mathrm{~mm}$. The VSWR is less than 1.15 up to the frequency range of $200 \mathrm{MHz}$. Model (d) has a similar configuration as that adapted for the lumped circuit model except that the axial length is divided into 10 sections and the outer diameter follows Eq. 2 along the axial direction for a lumped circuit model. It is very difficult to designate a boundary condition for the inner conductor due to the skin depth effect so that the permittivity of the water is not considered in the lumped circuit model. This means that the lumped 


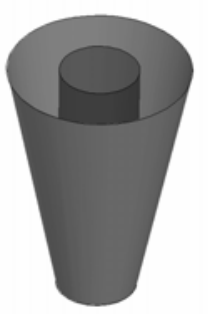

(a)

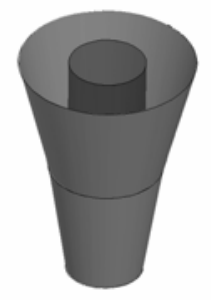

(c)

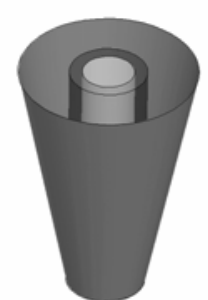

(b)

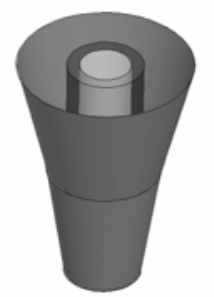

(d)
Fig. 3. Various models for a soda dummy load; an inner conductor is full of water and an outer conductor has a tapered structure (a), a tapered structure and the inner conductor is partially filled with water (b), a 2 step tapered structure and the inner conductor is full of water (c), a 2 step tapered structure and the inner conductor is partially filled with water (d); The white colored volume is the air and the blue colored one is the soda water

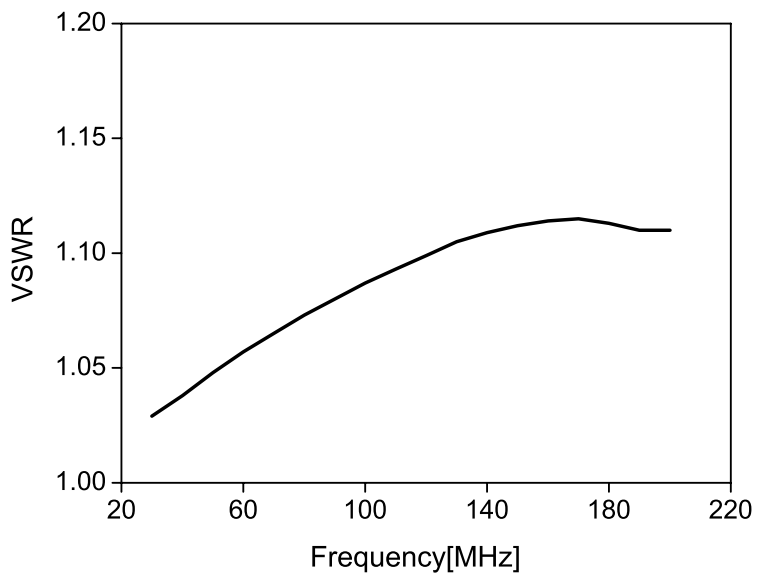

Fig. 4. VSWR vs. frequency using a lumped circuit model

circuit model is only effective when the skin depth is larger than the thickness of the soda water column.

The analyses for various models depicted in Fig. 3 are also performed by a numerical method using commercial software and their results are shown in Fig. 5. When the inner conductor is partially full of water, the two step conical shape (d) shows a low VSWR. However, when the inner conductor is fully filled with water as described in model (a) and (c), model (a) where the radius of the outer conductor is a form of the monotonic decreasing function shows a low VSWR. It seems that the shape function of the outer conductor is not so important in actual soda dummy load where the inner part of center conductor is full of water.

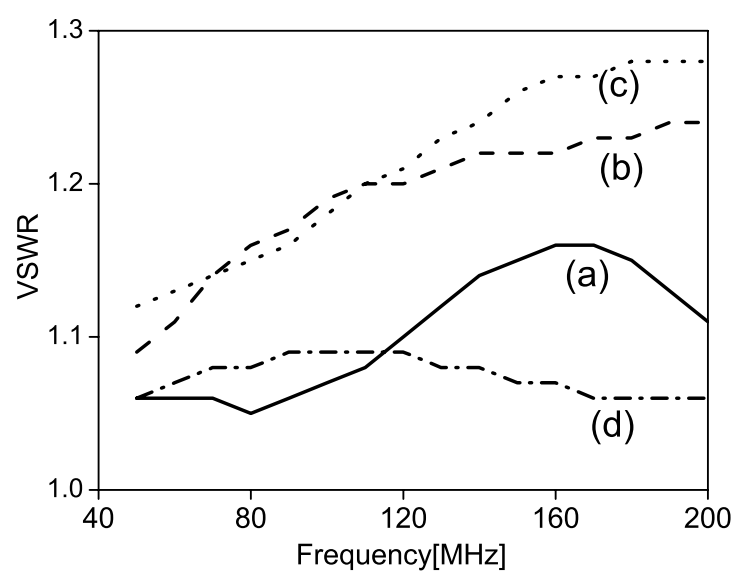

Fig. 5. VSWR vs. frequency using a numerical analysis for various models described in Fig. 3

\section{Thermal and Mechanical Design View- point}

The critical condition for the size of a dummy load is the capability of a water cooling for the $2 \mathrm{MW}$ heat generated on the water and the conductivity of soda water has a negative temperature coefficient. So it is crucial to maintain the temperature of soda water within $20^{\circ} \mathrm{C}$ for low VSWR of the dummy load. The flow rate is given by

$$
U=\frac{q}{c_{p} \Delta T_{b} \rho} \ldots \ldots \ldots \ldots \ldots \ldots \ldots \ldots \ldots \ldots
$$

where $c_{p}$ is the specific heat of the soda water, $q$ is the generated heat, $\rho$ is the density of the soda water and $\Delta T_{b}$ is the bulk temperature rise of the soda water. When we consider the typical case $\left(\Delta T_{b}\right.$ is $20^{\circ} \mathrm{C}$ and $q$ is $2 \mathrm{MW})$, the flow rate is about $0.02 \mathrm{~m}^{3} / \mathrm{sec}$. The pressure drop along the dummy load is dependent on the gap distance between the two guiding insulator cylinders. So it is necessary to have a gap distance between two insulator cylinders larger than $15 \mathrm{~mm}$ to allow to the cooling water to flow along for a distance of $50 \mathrm{~cm}$ without a large pressure drop of the water. Another point to consider is the selection of the insulator material used for guiding the water. It depends on the loss tangent values and the heat conductivity of the insulator material. The most promising material is alumina tube but it has much difficulty in shaping the structure, so poly-prophylene is used. Presently, a 2 MW soda water dummy load is being assembled.

\section{Summary}

The basic electrical design feature of a 2 MW soda water dummy load for a HF transmitter is investigated. The RF characteristics of the soda water dummy load are deduced using a lumped circuit model and a numerical analysis using commercial software. A lumped circuit model is only effective when the skin depth is larger than the thickness of soda water column. From the numerical analysis, the dummy load whose inner conductor is fully filled with soda water shows a low VSWR when its outer radius is a form of the monotonic decreasing 
function below $120 \mathrm{MHz}$.

\section{Acknowledgment}

The authors would like to thank Prof. T. Mutoh at NIFS for his helpful advice on the initial design of dummy load.

(Manuscript received Nov. 15, 2004)

\section{References}

(1) P.L.D. Abrie: The design of impedance-matching networks for radio-frequency and microwave amplifiers, Ch.2, Artech house Inc, Dedham (1985)

(2) R.S. Elliott: An introduction to guided waves and microwave circuits, p.231, Prentice Hall, New Jersey (1993)

Jong-Gu Kwak (Non-member) was born in Korea, on Dec.

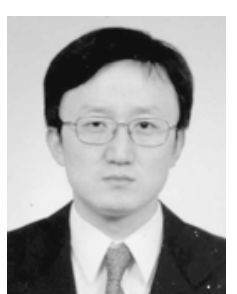
16, 1966. He received a $\mathrm{Ph}$. D. degree in nuclear engineering from Seoul National University in 1996, and is presently a senior researcher at Korea Atomic Energy Research Institute. He has worked on the low temperature plasma sources, and development of ICRF system for fusion research.
Song-Jong Wang (Non-member) was born in Korea, on

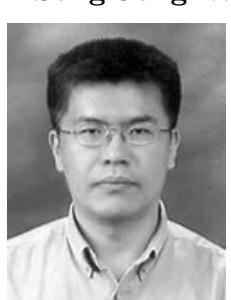
June 10, 1969. He received a $\mathrm{Ph}$. D. degree in physics from Soongsil University in 2003, and is presently a researcher at Korea Atomic Energy Research Institute. He has worked on RF plasma sources, and development of ICRF system for fusion research. Korean Physical Society, American Physical Society member.

Jae-Sung Yoon (Non-member) was born in Korea. $\mathrm{He}$

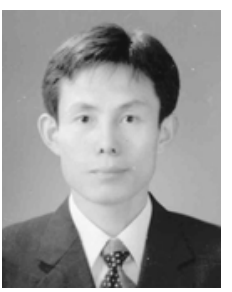
received a Master degree in microwave engineering from Chungnam National University in 2002, and is presently a principal researcher at Korea Atomic Energy Institute. He has worked on ICRF system. Korean Physical Society member.

Bong-Guen Hong (Non-member) was born in Korea. He

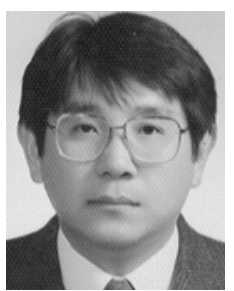
received a $\mathrm{Ph}$. D. degree in physics from Korea Advanced Institute of Science and Technology in 1987 , and is presently a principal researcher at Korea Atomic Energy Research Institute. He has worked on ICRF system. Korean Physical Society, Korean Nuclear Society member. 